\title{
Mechanism of Intrinsic Chemiluminescence Production from the Degradation of Persistent Chlorinated Phenols by the Fenton System: A Structure-Activity Relationship Study and the Critical Role of Quinoid and Semiquinone Radical Intermediates
}

\author{
Hui-Ying Gao, ${ }^{\dagger}, \mathrm{Li} \mathrm{Mao}^{\dagger}{ }^{\dagger}$ Feng Li, ${ }^{\dagger}$ Lin-Na Xie, ${ }^{\dagger}$ Chun-Hua Huang, ${ }^{\dagger}$ Jie Shao, ${ }^{\dagger}$ Bo Shao, ${ }^{\dagger}$ \\ Balaraman Kalyanaraman, ${ }^{\text {II }}$ and Ben-Zhan $\mathrm{Zhu}^{*}, \dagger, \S_{0}$ \\ ${ }^{\dagger}$ State Key Laboratory of Environmental Chemistry and Ecotoxicology, Research Center for Eco-Environmental Sciences and \\ University of Chinese Academy of Sciences, Chinese Academy of Sciences, Beijing, P.R. China 100085 \\ ${ }^{\ddagger}$ Science and Technology College, North China Electric Power University, Baoding, P.R. China 071051 \\ ${ }^{I}$ Department of Biophysics, Medical College of Wisconsin, Milwaukee, Wisconsin 53226, United States \\ ${ }^{\S}$ Linus Pauling Institute, Oregon State University, Corvallis, Oregon 97331, United States
}

Supporting Information

ABSTRACT: We found recently that intrinsic chemiluminescence $(\mathrm{CL})$ could be produced by all 19 chlorophenolic persistent organic pollutants during environmentally friendly advanced oxidation processes. However, the underlying mechanism for the structure-activity relationship (SAR, i.e., the chemical structures and the CL generation) remains unclear. In this study, we found that, for all 19 chlorophenol congeners tested, the CL increased with an increasing number of chlorine atoms in general; and for chlorophenol isomers (such as the 6 trichlorophenols), the CL decreased in the order of meta- > ortho-/para-Cl-substituents with respect to the $-\mathrm{OH}$ group of chlorophenols. Further studies showed that not only chlorinated quinoid intermediates but also, more interestingly, chlorinated

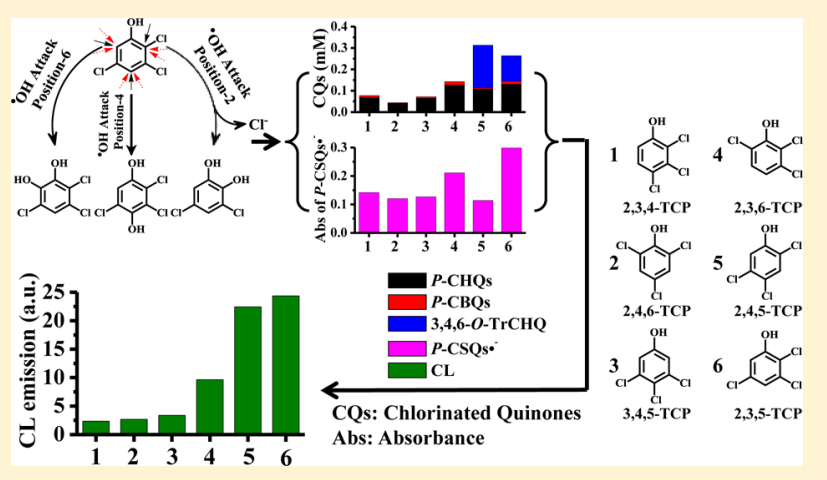
semiquinone radicals were produced during the degradation of trichlorophenols by the Fenton reagent; and the type and yield of which were determined by the directing effects, hydrogen bonding, and steric hindrance effect of the OH- and/or Cl-substitution groups. More importantly, a good correlation was observed between the formation of these quinoid intermediates and CL generation, which could fully explain the above SAR findings. This represents the first report on the structure-activity relationship study and the critical role of quinoid and semiquinone radical intermediates, which may have broad chemical and environmental implications for future studies on remediation of other halogenated persistent organic pollutants by advanced oxidation processes.

\section{INTRODUCTION}

Chlorophenols (CPs) have found wide use in pesticides, herbicides, disinfectants, wood preservatives, personal care formulations, dyestuff intermediates, and many other products, and they are also substantial byproducts of wood pulp bleaching with chlorine. ${ }^{1-3}$ CPs are poorly biodegradable with a half-life that can exceed some years in organic sediments. However, growing knowledge about the toxicities and environmental fates of specific chlorinated phenols has caused governments to regulate these compounds. ${ }^{2,4}$ Chlorinated phenols have been found in at least 166 of the 1467 National Priorities List sites, and 4 of them are listed by the U.S. Environmental Protection Agency (EPA) as priority pollutants, including 2-chlorophenol (2-CP), 2,4-dichlorophenol (2,4DCP), 2,4,6-trichlorophenol (2,4,6-TCP), and pentachlorophenol (PCP), which are present in the environment in significant quantities. ${ }^{1,5,6}$ Moreover, except for 2-CP, 2 other chlorophenols, 2,4,5-trichlorophenol (2,4,5-TCP) and 2,3,4,6-tetrachlorophenol $(2,3,4,6-\mathrm{TeCP})$, have been classified as group $2 \mathrm{~B}$ environmental carcinogens by the International Association for Research on Cancer (IARC). ${ }^{6,7}$ Therefore, it is necessary to develop highly efficient and reliable technologies for their removal.

Although there are several ways to produce hydroxyl radicals $\left({ }^{\bullet} \mathrm{OH}\right)$, the Fenton reaction, as one of the advanced oxidation processes (AOPs) for the treatment of wastewaters containing chlorophenols, ${ }^{4}$ draws the most attention because it is fast, low

Received: September 14, 2016

Revised: December 28, 2016

Accepted: January 26, 2017

Published: January 27, 2017 
cost, easy to operate at room temperature, and environmentally benign. 8,9

Chemiluminescence (CL) is a phenomenon in which molecules in a chemically generated excited state liberate energy $\left(>45 \mathrm{kcal} \cdot \mathrm{mol}^{-1}\right)$ with light emission. It has been found that $\mathrm{CL}$ often accompanies decomposition of organic peroxides and generation of free radical intermediates. ${ }^{10-13}$ In recent years, the CL method has become an attractive analytical tool due to its numerous advantages such as inherently highly sensitive, rapid, and simple to operate, wide dynamic range, and low cost. Many important CL systems, such as luminol, lucigenin, potassium permanganate, and cerium(IV), have been explored for the analysis of organic and inorganic species in various research fields, including the applications in pharmaceuticals, biomolecules, antioxidants, pesticides, and environmental water. ${ }^{14-16}$

During our study of metal-independent hydroxyl/alkoxyl/ ketoxy radical production by halogenated quinones and $\mathrm{H}_{2} \mathrm{O}_{2}$ (or organic hydroperoxides) ${ }^{17-23}$ and their potential biological effects, ${ }^{24-26}$ we found that unprecedented hydroxyl radicaldependent two-step chemiluminescence could be generated by polyhalogenated quinones and $\mathrm{H}_{2} \mathrm{O}_{2}{ }^{20}$ Recently, we also observed an intrinsic CL generation by all 19 chlorophenols (which are the parent compounds of chlorinated quinones) and the classic ${ }^{\bullet} \mathrm{OH}$-generating Fenton system, even in the absence of fluorescent agents. ${ }^{27}$ Interestingly and unexpectedly, the CL emission from the chlorinated phenols was found to depend not only on the chlorination level but also on the chlorine substitution position. ${ }^{27}$ However, it remains unclear what is the accurate structure-activity relationship (SAR) between the chemical structures and CL generation.

Therefore, in this study, we addressed the following questions: (i) Is there any SAR between the chemical structures of CPs and CL generation; and if so, what is the accurate SAR; (ii) Whether chlorinated quinoid and/or semiquinone radical intermediates are produced during the degradation of CPs by the Fenton system; (iii) Are there any correlations between the yields and types of these quinoid and/or semiquinone radical intermediates and CL production; (iv) What is the underlying molecular basis for this correlation.

\section{MATERIALS AND METHODS}

Chemiluminescence (CL) Analysis. The CL produced by $\mathrm{CPs}$ and the Fenton reagent was measured by an ultraweak $\mathrm{CL}$ analyzer (Institute of Biophysics, Chinese Academy of Science, China) with a CR-120 red-sensitive photomultiplier tube (PMT, Hamamatsu, Japan); the CL analyzer was operated in pulse mode. In order to eliminate the effect of dissolved oxygen and $\mathrm{CO}_{2}$ of solution, we conducted the $\mathrm{CL}$ experiments through aeration of argon in the phosphate buffer. We found that neither dissolved oxygen nor $\mathrm{CO}_{2}$ showed any effect on $\mathrm{CL}$ in this system, even for a long aerating time. Thus, it was not necessary to pretreat the phosphate buffer before CL experiments in this system. The CL determination was carried out in a $3 \mathrm{~mL}$ glass cuvette and started by the injection of $\mathrm{Fe}^{2+}$ EDTA $^{28-30}$ after CP solution was mixed with $\mathrm{H}_{2} \mathrm{O}_{2}$. The CL signal was recorded by a computer equipped with a dataacquisition interface. Data acquisition and treatment were performed with BPCL software. The total intensity of CL was integrated during the whole process for CL measurements. The CL emission spectrum was obtained using a set of interference filters, with the wavelengths from 400 to $640 \mathrm{~nm}$, which were placed between the sample cuvette and the photomultiplier tube. During the determination of CL emission wavelength, appropriate corrections were applied for both spectral response of the photomultiplier tube and transmissivity of filters. However, the CL emission spectrum obtained from the CL analyzer could only provide an approximate range; therefore, a spectrofluorometer (Varian) set at CL mode was used to obtain a continuous CL spectrum and get a more accurate maximum wavelength. Each point of CL analysis on the figures and tables represents the mean value from 3 to 6 assays in parallel. The total CL emission in this CPs/Fenton system shows good reproducibility, within a precision of $\pm 5.0 \%$ for repetitive measurements. Meanwhile, in order to make the figures clearer, each point on the figures and tables represents the mean value from 3 to 6 assays in parallel.

Detection and Identification of Chlorinated $p$-Semiquinone Radicals. The spectra and kinetics of chloro- $p$ semiquinone radicals $\left(P\right.$-CSQ $\left.s^{\bullet}\right)$ formation by trichlorophenols (TCPs) and the Fenton reagent were recorded and monitored by complementary application of both UV-visible spectrometric methods coupled with stopped-flow technique and direct ESR. Each plot of these analyses on P-CSQs ${ }^{\bullet}$ was obtained from 3 assays in parallel, and each point on the figures and tables represents the mean value from these corresponding assays.

i. Electron Spin Resonance (ESR) Studies. ESR spectra were recorded either immediately after the interaction between CPs and the Fenton reagent or at indicated time intervals on a Bruker EMX-plus spectrometer operating at $9.84 \mathrm{GHz}$ and a cavity equipped with a Bruker Aquax liquid sample cell. Typical spectrometer parameters were as follows: scan range, $100 \mathrm{G}$; field set, $3513 \mathrm{G}$; time constant, $82 \mathrm{~ms}$; scan time, $82 \mathrm{~s}$; modulation amplitude, $1 \mathrm{G}$; modulation frequency, $100 \mathrm{kHz}$; receiver gain, $1.00 \times 10^{5}$; and microwave power, $20 \mathrm{~mW}$. The hyperfine splitting constants were measured by using the simulation software WinSim (version 0.96) (NIEHS). ${ }^{31}$

ii. UV-Visible Spectral Analysis. UV-visible spectra of the interaction between CPs and the Fenton reagent were monitored by a UV-visible spectrophotometer (Beckman DU-800) in the Chelex-treated phosphate buffer (0.1 M, pH 7.4) at room temperature. $P$-CSQs ${ }^{\bullet}$ were obtained from the reaction between corresponding chloro- $p$-benzoquinones $(P$ CBQs) and ascorbic acid (Vc) at a 1:1 molar ratio, ${ }^{32-34}$ and the spectra were recorded from 400 to $800 \mathrm{~nm}$. The kinetics of $P$ $\mathrm{CSQs}^{\bullet}$ formation by the TCPs/Fenton system was followed at the corresponding characteristic wavelength.

iii. Stopped-Flow Kinetic Analysis. Stopped-flow spectra were collected on a Biologic SFM-300 system equipped with three syringes and were capable of sequential mixing, with a high-speed diode array detector. Syringe 1 was filled with the solution of TCPs in the phosphate buffer (0.1 M, pH 7.4), while syringe 2 was filled with an aqueous solution containing $\mathrm{H}_{2} \mathrm{O}_{2}$. The solutions of the above two syringes were premixed before rapidly mixing with syringe 3 of $\mathrm{Fe}^{2+}$-EDTA solution, and the syringe volume ratio for the three reactants was $1: 1: 1$. The resulting reactant mixture was introduced into the optical cell where the transient change in absorbance was measured. The stopped-flow spectra were recorded from 400 to $600 \mathrm{~nm}$, and the kinetics of $\mathrm{P}$-CSQs ${ }^{\bullet}$ formation by the TCPs/Fenton system was monitored at the corresponding characteristic wavelength. 


\section{RESULTS AND DISCUSSION}

Intrinsic CL Can Be Produced by All 19 CPs and the Fenton Reagent, and the CL Intensity Produced by the CPs/Fenton Reagent Was Dependent on Not Only the Number but Also the Position of Chlorination. As mentioned above, we found recently that intrinsic CL could be produced by all 19 chlorophenol congeners and the Fenton reagent, which was further confirmed under different experimental conditions in this study (Figure S1). Interestingly, we found that the CL intensity of CPs in the Fenton system was not only correlated to its chlorination level but also to the spatial distribution of the chloro-substitution. The following SAR between the chemical structures of CPs and CL generation was observed: (i) the CL intensity increases with an increasing number of chloro-substitution; (ii) among the CPs isomers, CPs with position-2, -3 , or -5 chloro-substitution (which are considered stimulative sites) exhibit stronger CL, whereas those with position- 4 or -6 chloro-substitution (inhibitory sites) produce relatively weaker CL.

However, there are some notable exceptions for the two general trends. Take the six trichlorophenols (TCPs) for example, the CL intensity of 2,3,4-trichlorophenol (2,3,4-TCP) with only one inhibitory site is weaker than $2,4,6$-TCP with both 4- and 6-inhibitory sites.

The SAR for CL was not only a special phenomenon under one specific experimental condition, analogous results were also observed under various different experimental conditions, which further confirmed the above findings. Therefore, it is interesting to know why such a SAR for CL exists, and what is the underlying molecular mechanism.

Among the five isomeric groups ( 3 monochlorophenols, 6 dichlorophenols, 6 trichlorophenols, 3 tetrachlorophenols, and 1 pentachlorophenol) of all 19 chlorophenol congeners, both trichlorophenols (TCPs) and dichlorophenols have 6 isomers, but TCPs produced much stronger CL emission as compared to dichlorophenols. Therefore, to better understand the SAR for CL generation and its underlying molecular mechanism, 6 TCP isomers were chosen as model chlorophenols for more detailed investigations.

We found that the intensity of CL emission was dependent on the concentrations of both TCPs and the Fenton reagent (Figure 1A-D). The CL produced by the TCPs/Fenton system was also found to depend on $\mathrm{pH}$ of the buffer: No CL was observed at $\mathrm{pH} \leq 5$; as the $\mathrm{pH}$ increased, the intensity of $\mathrm{CL}$ increased progressively and reached a maximum at $\mathrm{pH}$ 7.4-8; a further increase of $\mathrm{pH}$, however, led to a decline of CL intensity (Figure 1E). We also found that the CL intensity was temperature-dependent: the higher the temperature in the range of 20 to $80{ }^{\circ} \mathrm{C}$, the stronger the CL intensity (Figure $1 \mathrm{~F}$ ). The CL emission spectra of the TCPs/Fenton system were obtained by both an ultraweak CL analyzer and a fluorospectrometer, respectively (Figure $1 \mathrm{G}$ and $1 \mathrm{H}$ ). Analogous CL emission spectra were observed for these three chlorinated phenols, with the maximum CL emission at a broad band of 535-555 $\mathrm{nm}$.

Among the six TCP isomers tested, 2,3,5-trichlorophenol $(2,3,5-\mathrm{TCP})$ produced the strongest CL. The CL produced by 2,3,5-TCP in the Fenton system was found to be directly dependent on ${ }^{\circ} \mathrm{OH}$ formation, as indicated by the following lines of evidence: (i) The CL produced by the TCPs/Fenton system was markedly inhibited by several typical ${ }^{\circ} \mathrm{OH}$ scavengers, such as dimethyl sulfoxide (DMSO), L-cysteine,
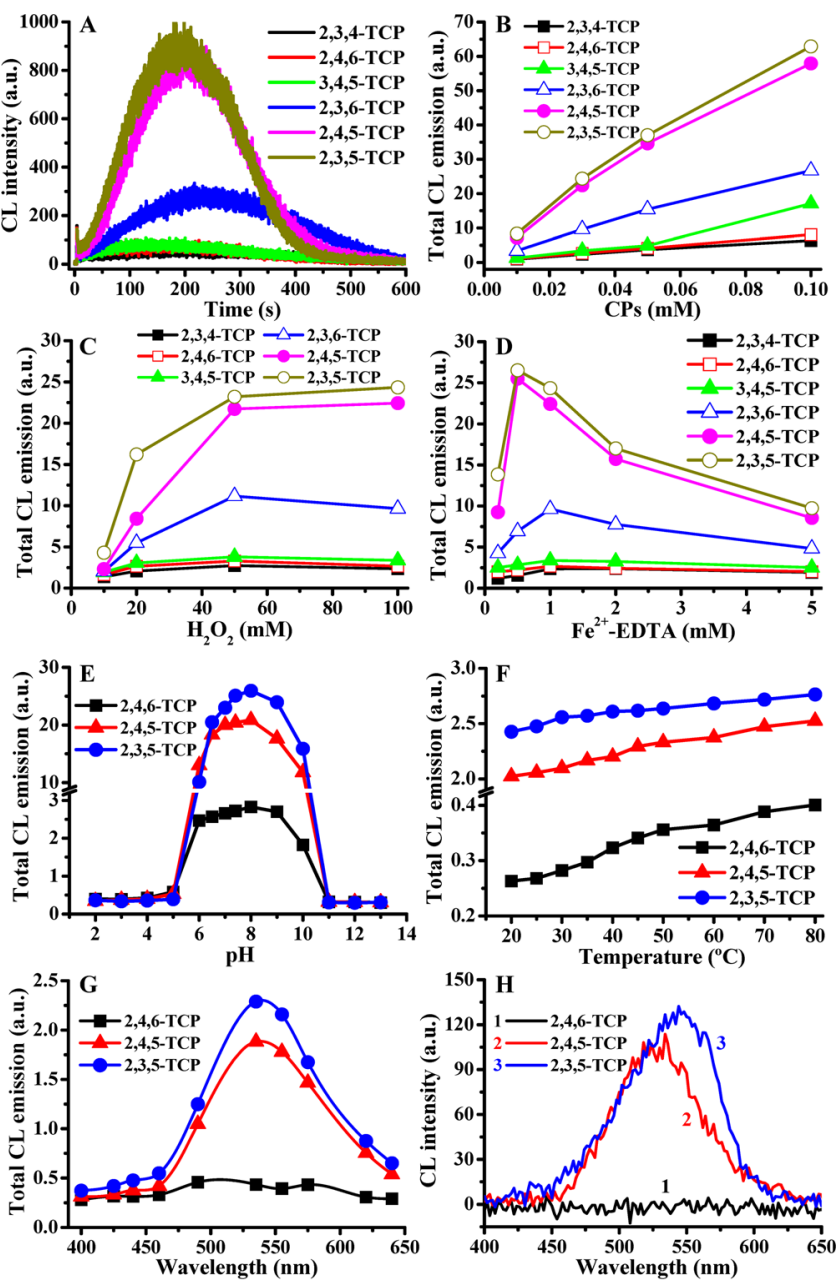

Figure 1. The CL emission could be produced during the degradation of TCPs by the Fenton system. (A) CL could be produced by the TCPs/Fenton system. TCPs, 0.03 mM; $\mathrm{H}_{2} \mathrm{O}_{2}, 100 \mathrm{mM} ; \mathrm{Fe}^{2+}$-EDTA, $1 \mathrm{mM}$. (B-D) The dose-dependent effects of chlorophenols, $\mathrm{H}_{2} \mathrm{O}_{2}$, and $\mathrm{Fe}^{2+}$-EDTA on CL production by chlorophenols/Fenton. TCPs, 0.01-0.1 mM; $\mathrm{H}_{2} \mathrm{O}_{2}, 10-100 \mathrm{mM}$; Fe ${ }^{2+}$-EDTA, 0.2-5 mM. (E and F) The effect of $\mathrm{pH}$ and temperature on CL production by 2,3,5-/ 2,4,5-/2,4,6-TCP and the Fenton reagent. 2,3,5-/2,4,5-/2,4,6-TCP, $0.03 \mathrm{mM} ; \mathrm{H}_{2} \mathrm{O}_{2}, 100 \mathrm{mM}$; Fe ${ }^{2+}$-EDTA, $1 \mathrm{mM}$. (G) The CL emission spectra measured by static-injection ultraweak $\mathrm{CL}$ analysis. 2,3,5-/ 2,4,5-/2,4,6-TCP, 0.03 mM; $\mathrm{H}_{2} \mathrm{O}_{2}, 100 \mathrm{mM} ; \mathrm{Fe}^{2+}$-EDTA, $1 \mathrm{mM} .(\mathrm{H})$ The $\mathrm{CL}$ emission spectra measured by spectrofluorometry. 2,3,5-/ 2,4,5-/2,4,6-TCP, 2 mM; $\mathrm{H}_{2} \mathrm{O}_{2}, 1000 \mathrm{mM}$; $\mathrm{Fe}^{2+}$-EDTA, $5 \mathrm{mM}$. All reactions were carried out in the chelex-pretreated phosphate buffer (0.1 M, pH 7.4).

and benzoate (Figure S2A), and the concentrations for each - $\mathrm{OH}$ scavenger to inhibit $50 \%$ of CL were found to be 0.48 , 0.30 , and $0.17 \mathrm{mM}$, respectively; (ii) not only the CL reaction and ${ }^{\circ} \mathrm{OH}$-generation could be markedly accelerated but also the $\mathrm{CL}$ intensity and ${ }^{\bullet} \mathrm{OH}$ yield were enhanced significantly as the concentration of $\mathrm{H}_{2} \mathrm{O}_{2}$ was increased (Figure $1 \mathrm{C}$ and Figure $\mathrm{S} 5 \mathrm{E})$; (iii) $\mathrm{CL}$ was also observed when $\mathrm{Fe}^{2+}$-EDTA was substituted by two other well-known ${ }^{\bullet} \mathrm{OH}$-generating Fenton agents $\mathrm{Fe}^{2+}$-DTPA (diethylenetriaminepentaacetic acid) and $\mathrm{Fe}^{2+}$-NTA (nitrilotriacetic acid) (Figure S2B); ${ }^{35}$ and (iv) CL could be produced not only with the classic $\mathrm{Fe}^{2+}$-mediated Fenton systems but also with other redox-active metalmediated Fenton-like systems including $\mathrm{Co}(\mathrm{II})-\mathrm{EDTA} / \mathrm{H}_{2} \mathrm{O}_{2}$, $\mathrm{Cr}(\mathrm{III})-\mathrm{EDTA} / \mathrm{H}_{2} \mathrm{O}_{2}$, and $\mathrm{VO}(\mathrm{II})-\mathrm{EDTA} / \mathrm{H}_{2} \mathrm{O}_{2}$, which could 


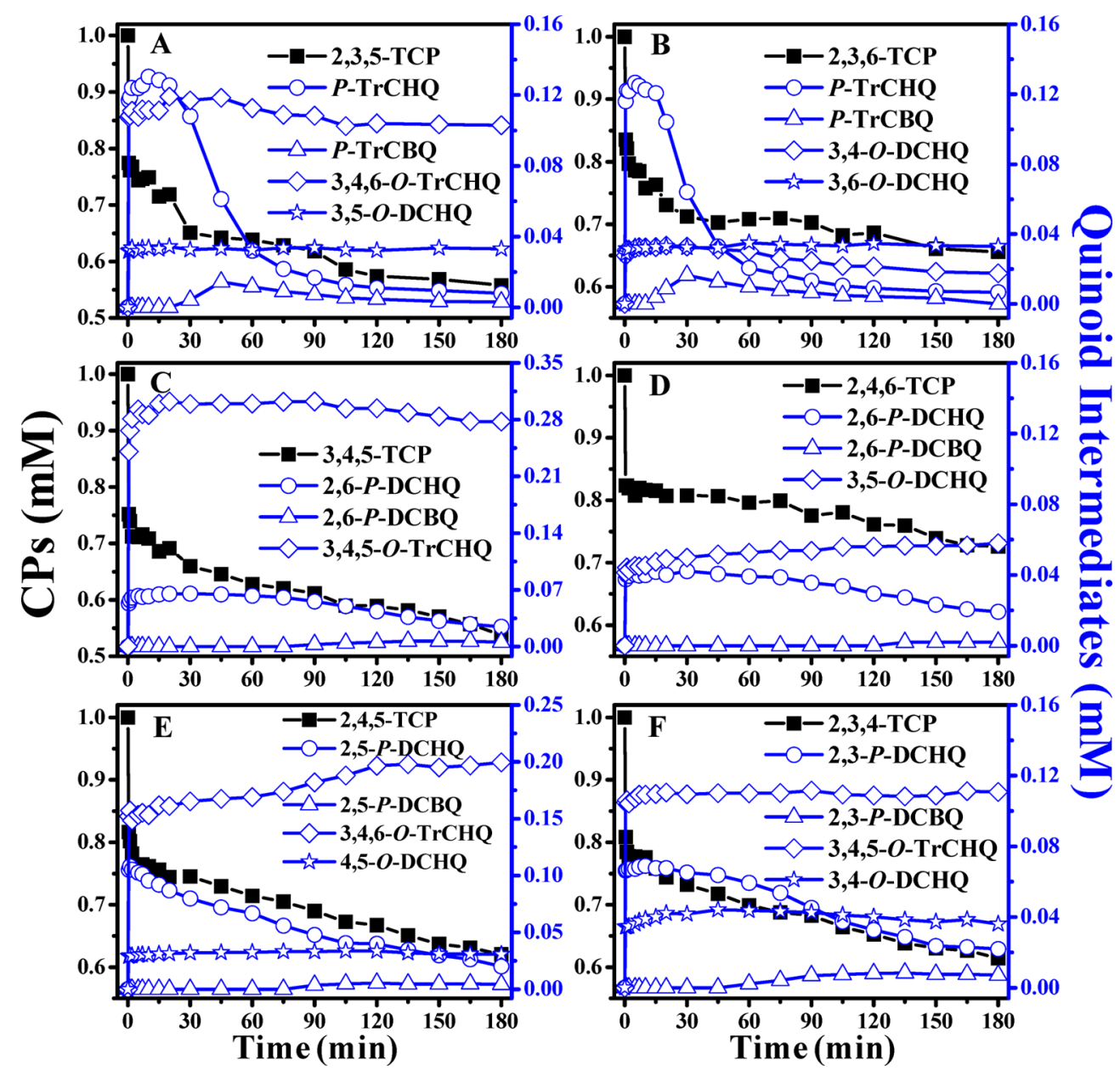

Figure 2. Time course of the degradation of parent compounds trichlorophenols ((A) 2,3,5-TCP; (B) 2,3,6-TCP; (C) 3,4,5-TCP; (D) 2,4,6-TCP; (E) 2,4,5-TCP; (F) 2,3,4-TCP) and the formation of the main intermediates P-CHQs, $P$-CBQs, and O-CHQs by HPLC analysis. TCPs, 1 mM; $\mathrm{H}_{2} \mathrm{O}_{2}, 1 \mathrm{mM}$; Fe ${ }^{2+}$-EDTA, $3 \mathrm{mM}$. All reactions were carried out in the chelex-pretreated phosphate buffer $(0.1 \mathrm{M}, \mathrm{pH} 7.4)$ at $25{ }^{\circ} \mathrm{C}$.

all produce ${ }^{\bullet} \mathrm{OH}$ (Figure S2C). Interestingly, the $\mathrm{CL}$ trend of TCPs in all these ${ }^{\bullet} \mathrm{OH}$-generating systems was exactly identical to the classic Fenton system.

Chlorinated Quinoid Compounds Were Found as the Major Reaction Intermediates during the Degradation of TCPs by the Fenton Reagent. To further investigate the underlying mechanism of the above interesting findings, the time course of CL emission and degradation of TCPs (here we choose 2,3,5-/2,4,5-TCP as two examples) by the Fenton system were investigated, and we found that the CL emission could not reach maximum until 2,3,5-TCP (or 2,4,5-TCP) was almost completely degraded (more than 90\%) (Figure S3). These results suggested that the species responsible for CL emission should not be TCPs itself but probably its degradation intermediates.

It has been shown that $\mathrm{CPs}$ can be oxidized by ${ }^{\bullet} \mathrm{OH}$ during advanced oxidation processes (AOPs) to produce several chlorinated quinoid intermediates, which include chloro- $p$ hydroquinones ( $P$-CHQs), chloro- $p$-benzoquinones ( $P$-CBQs), chloro-o-hydroquinones ( $\mathrm{O}$-CHQs), and chloro-o-benzoquinones $(O-C B Q s) .{ }^{27,36}$ We found that this is also the case in our study. The major quinoid intermediates produced by TCPs were identified by the HPLC-UV method with the respective authentic compounds as standards. As expected, we found that three of these four chlorinated quinoid compounds, including $P$-CHQs, $P$-CBQs, and $\mathrm{O}$-CHQs, were indeed the major quinoid degradation intermediates of TCPs (Figure 2). For example, 2,3,5-TCP could produce trichloro- $p$-benzoquinone (P-TrCBQ), trichloro- $p$-hydroquinone (P-TrCHQ), 3,4,6trichloro-o-hydroquinone (3,4,6-O-TrCHQ), and 3,5-dichloro$o$-hydroquinone (3,5-O-DCHQ); while 2,4,5-TCP could produce 2,5-dichloro- $p$-benzoquinone (2,5-P-DCBQ), 2,5dichloro- $p$-hydroquinone (2,5-P-DCHQ), 3,4,6-O-TrCHQ and 4,5-dichloro-o-hydroquinone (4,5-O-DCHQ); and 2,4,6TCP could produce 2,6-dichloro- $p$-benzoquinone (2,6-PDCBQ), 2,6-dichloro-p-hydroquinone (2,6-P-DCHQ), and 3,5-dichloro-o-hydroquinone (3,5-O-DCHQ). However, OCBQs were not detected, possibly due to their extreme instability. Based on the formation of the same chlorinated quinoid compounds $P$-CHQs/P-CBQs, the six TCPs can be divided into four different groups. One group includes 2,3,5and 2,3,6-TCP, which could produce $P$-TrCHQ/P-TrCBQ; the second group includes 3,4,5-trichlorophenol (3,4,5-TCP) and 2,4,6-TCP, which could produce 2,6-P-DCHQ/2,6-P-DCBQ the other two groups include 2,4,5-TCP and 2,3,4-TCP, which could produce 2,5-P-DCHQ/2,5-P-DCBQ and 2,3-P-DCHQ/ $2,3-P-D C B Q$ respectively. Interestingly, the formation kinetics of these major quinoid intermediates were found to be well correlated with that for the degradation of TCPs (Figure 2).

A Good Correlation Was Observed between CL Emission and the Formation of the Chlorinated Quinoid Intermediates. We found recently that CL could be produced 
by several kinds of $P-\mathrm{CBQ}$ and $\mathrm{O}-\mathrm{CHQ}$ in the Fenton system. In the present study, we also found the following, even under various different experimental conditions: (i) the CL emission of $P$-CBQs increased with an increasing number of chlorine atoms on the quinone ring (i.e., $p$-benzoquinone $(P$-BQ $<$ chloro- $p$-benzoquinone $(P$-CBQ $)<$ dichloro- $p$-benzoquinone isomers ( $P$-DCBQs $)<P$-TrCBQ $<$ tetrachloro- $p$-benzoquinone (P-TCBQ); (ii) among the three dichloro- $p$-benzoquinone isomers ( $P$-DCBQs), the order of $\mathrm{CL}$ emission is 2,3-P$\mathrm{DCBQ}<2,6-P$-DCBQ $<2,5-P-\mathrm{DCBQ}$ (iii) the $\mathrm{CL}$ emissions were especially strong for 3,4,6-O-TrCHQ but much weaker for other $\mathrm{O}$-CHQs (Figure 3). Therefore, we speculated that the
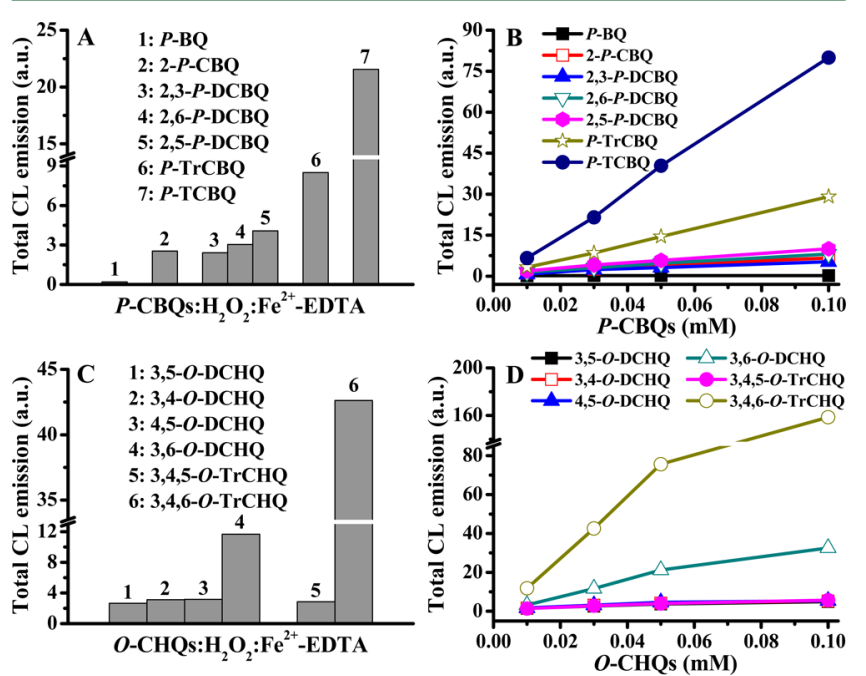

Figure 3. $\mathrm{CL}$ emission from the major chlorinated quinoid compounds $P$-CBQs and $O$-CHQs in the Fenton system. (A and C) The trend of $\mathrm{CL}$ emission from $P$-CBQs and $O-C H Q$ s. $P$-CBQs and $O$-CHQs, $0.03 \mathrm{mM} ; \mathrm{H}_{2} \mathrm{O}_{2}, 100 \mathrm{mM}$; Fe ${ }^{2+}$-EDTA, $1 \mathrm{mM}$. (B and D) The dose-dependent effect of $P$-CBQs and $O-C H Q s$ on $C L$ emission. $P$-CBQs and $O$-CHQs, 0.01-0.1 mM; $\mathrm{H}_{2} \mathrm{O}_{2}, 100 \mathrm{mM}$; Fe ${ }^{2+}$-EDTA, 1 $\mathrm{mM}$. All reactions were carried out in the chelex-pretreated phosphate buffer $(0.1 \mathrm{M}, \mathrm{pH} 7.4)$ at $25^{\circ} \mathrm{C}$.

CL intensity of TCPs might be mainly dependent on the yields and types of the $P$-CBQs (since $P$-CHQs can also be further oxidized to form $P$-CBQs) and 3,4,6-O-TrCHQ. As discussed below, this was found to be indeed the case.

The maximum yields of each quinoid intermediate produced by TCPs were summarized in Table S1. We found that there is a good correlation between CL production and the total yields of their corresponding quinoid intermediates $P$-CHQs/P-CBQs and 3,4,6-O-TrCHQ especially for those $\mathrm{CPs}$ which could produce the same $P$-CHQs/P-CBQs intermediates (Figure 4).

Then the question is why such a correlation exists.

From the above studies, three general principles were observed on $\mathrm{CL}$ production from $\mathrm{P}-\mathrm{CBQ}$ s and $\mathrm{O}-\mathrm{CHQ}$ in the Fenton system: (i) Among the six P-CBQs, the higher the chloro-substitution, the stronger the CL emission; (ii) Among the $\mathrm{O}$-CHQs tested, 3,4,6-O-TrCHQ produced the strongest CL; (iii) For the same $P$-CBQ/P-CHQ and 3,4,6-O-TrCHQ, the higher yields of these chlorinated quinoid intermediates were produced, the stronger the $\mathrm{CL}$ emission. Interestingly, the SAR for CL can be well explained by the above three basic principles (Table S2).

For the first general trend, the CL intensity of CPs with different chloro-substitution increases in the following order: 4-

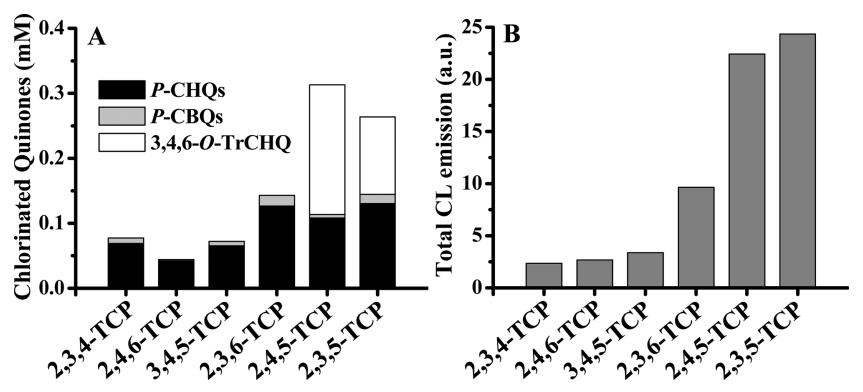

Figure 4. A good correlation was observed between the total yields of $P$-CHQs/P-CBQs and 3,4,6-O-TrCHQ and the CL emission produced by TCPs in the Fenton system. (A) The total yields of $P$ CHQs/P-CBQs and 3,4,6-O-TrCHQ. TCPs, $1 \mathrm{mM} ; \mathrm{H}_{2} \mathrm{O}_{2}, 1 \mathrm{mM}$; $\mathrm{Fe}^{2+}$-EDTA, $3 \mathrm{mM}$. (B) CL emission from TCPs. TCPs, $0.03 \mathrm{mM}$; $\mathrm{H}_{2} \mathrm{O}_{2}, 100 \mathrm{mM} ; \mathrm{Fe}^{2+}$-EDTA, $1 \mathrm{mM}$. The reactions were carried out in the chelex-pretreated phosphate buffer $(0.1 \mathrm{M}, \mathrm{pH} 7.4)$ at $25^{\circ} \mathrm{C}$.

CP $<2,4-\mathrm{DCP}<2,4,6-\mathrm{TCP}<2,3,4,6-\mathrm{TeCP}<\mathrm{PCP}$. This might be due to the fact that the $\mathrm{CL}$ emission by the major quinoid intermediates (P-BQ $P$-CBQ 2,6-P-DCBQ $P$-TrCBQ $P$ $T C B Q$ respectively) of these $C P s$ increase gradually as mentioned above.

For the second general trend, for the CPs isomers, CPs with position-2, -3 , or $-5 \mathrm{Cl}$-substitution (stimulative sites) exhibit stronger $\mathrm{CL}$, whereas those with position-4, or $-6 \mathrm{Cl}$ substitution (inhibitory sites) produce relatively weaker CL. For the six TCP isomers, the order of CL emission is 2,3,4TCP $<2,4,6$-TCP $<3,4,5$-TCP $<2,3,6-$ TCP $<2,4,5$-TCP $<$ 2,3,5-TCP. This may be attributed to the following fact: both $P$ TrCBQ and 3,4,6-O-TrCHQ were produced by 2,3,5-TCP (For the reaction of 2,3,5-TCP by ${ }^{\circ} \mathrm{OH}$ attack, see Scheme 1 . Dashed lines indicate possible pathways involving $\mathrm{O}-\mathrm{CBQs}$ which were not observed in this study).

Similar to the reaction of 2,3,5-TCP, 3,4,6-O-TrCHQ with high yield was produced by $2,4,5-\mathrm{TCP}$, whereas only $P$-TrCBQ was produced by 2,3,6-TCP; 2,6-P-DCBQ was produced by $3,4,5-\mathrm{TCP}$ with higher yield than that by 2,4,6-TCP; and 2,3-PDCBQ was produced by $2,3,4-T C P$, which is the weakest CL producer among the three $P$-DCBQ isomers. This should also explain why the CL emission produced by $2,3,4-\mathrm{TCP}$ is the weakest among the 6 TCPs.

Possible Molecular Basis for the Correlation between $\mathrm{CL}$ and Chlorinated Quinoid Intermediates. As mentioned above, we found that the CL generation from the TCPs/ Fenton system correlated well with the formation of different types of quinoid intermediates and their yields. Now the question is what is the underlying molecular basis for such SAR.

When ${ }^{\circ} \mathrm{OH}$ attacks $\mathrm{CPs}$, the types and yields of the chlorinated quinoid intermediates $(P-C H Q s / P-C B Q s$ and $O$ $\mathrm{CHQ}$ ) were determined by the basic physiochemical properties of CPs, which include the electron-withdrawing properties of $\mathrm{Cl}$ atoms and the directing effects of $-\mathrm{OH}$ and $-\mathrm{Cl}$ groups as listed below: (i) As the number of electron-withdrawing Clsubstituent increases, the $\pi$-electron density in aromatic ring decreases, and, as a result, it makes the phenolic ring less favorable for electrophilic attack by ${ }^{\bullet} \mathrm{OH}$; however, it makes the quinone ring (the oxidation intermediates of chlorophenols) more favorable for nucleophilic attack by $\mathrm{H}_{2} \mathrm{O}_{2} ;{ }^{18}$ (ii) Although both $-\mathrm{OH}$ and $-\mathrm{Cl}$ are ortho- and para-directing groups for further substitution or addition by ${ }^{\bullet} \mathrm{OH}$, the directing power by $-\mathrm{OH}$ is much stronger than $-\mathrm{Cl}^{37}$ thus, the types of $P$-CBQs/ $\mathrm{P}$-CHQs and $\mathrm{O}$-CHQs formation are mainly dependent on the 
Scheme 1

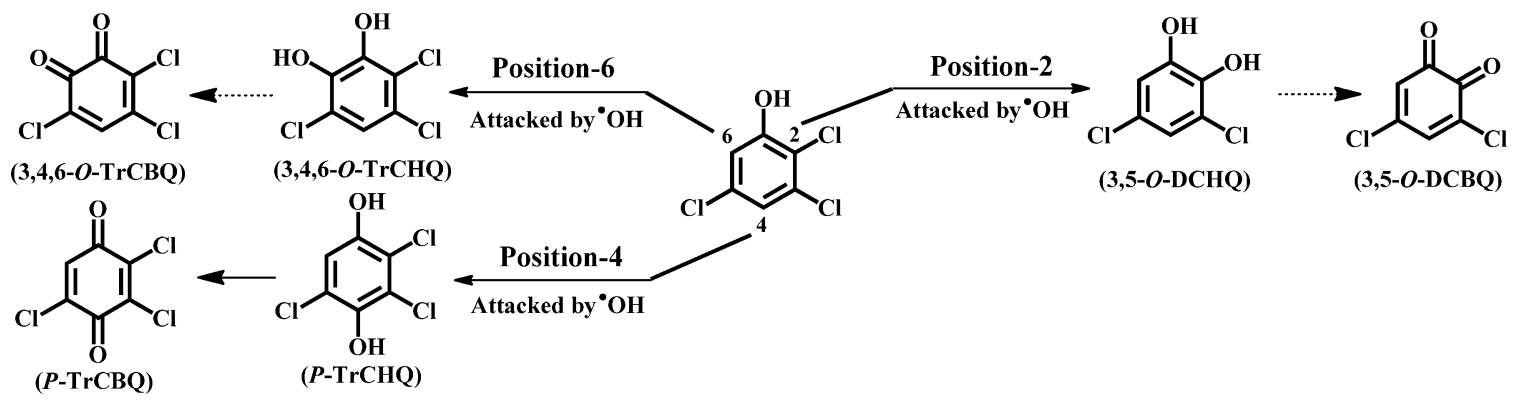

$-\mathrm{OH}$ group; (iii) When the chlorine substituents were at the meta positions of the phenolic ring, the directing effects of $-\mathrm{OH}$ and meta- $\mathrm{Cl}$ will reinforce each other, and the reactions with ${ }^{\bullet} \mathrm{OH}$ should be highly regioselective for ortho- and parapositions (more $P$-CBQs/P-CHQs should be formed); (iv) Meanwhile, for chlorophenol isomers, if more $-\mathrm{Cl}$ were at the meta positions of the phenolic ring, more highly chlorinated hydroquinones and catechols would be produced by the ${ }^{\bullet} \mathrm{OH}$ attack, since the dechlorination was less likely to occur; (v) - $\mathrm{OH}$ attacks more readily ortho- and para-positions not occupied by $-\mathrm{Cl}$ groups. It is well-known that the more negative charge density of the $\mathrm{C}$ atom on the aromatic ring, the more favorable the electrophilic ${ }^{\bullet} \mathrm{OH}$ would attack it. Density functional theory (DFT) calculations were conducted on the atomic polar tensors (APT) charge for TCPs with the B3LYP/ $6-311+G^{*}$ method. $^{38}$ The charge density of the free positions are indeed more negative than positions occupied by $-\mathrm{Cl}$ (Table S3); (vi) $\mathrm{Cl}$ at position-2 is very difficult to be attacked by ${ }^{\circ} \mathrm{OH}$, due to both steric hindrance effect ${ }^{39}$ and the intramolecular hydrogen bonding ${ }^{40}$ between $-\mathrm{OH}$ and $-\mathrm{Cl}$ groups; (vii) Even if the two ortho-positions were both substituted by $\mathrm{Cl}$ atoms, the steric hindrance effects of the $\mathrm{Cl}$ atom at position- 2 was always stronger than that at position- 6 based on IUPAC nomenclature rules. Thus, on the aromatic ring, the sites readily attacked by ${ }^{\circ} \mathrm{OH}$ are position- 4 and -6 , not position-2. The combination of the above seven factors should play decisive roles in SAR for CL produced by the CPs/ Fenton system.

To further illustrate the role of these factors in SAR for CL, three typical TCP isomers (2,3,5-, 2,4,5-, and 2,4,6-TCP) were taken as examples for detailed analysis. As shown in Scheme S1, the $-\mathrm{OH}$ group should determine the sites attacked by ${ }^{\bullet} \mathrm{OH}$ on the phenolic ring (noted as the solid arrows), while the meta- $\mathrm{Cl}$ groups should determine the reactivity of TCPs attacked by - $\mathrm{OH}$ (noted as the dashed arrows). In other words, the more the dashed arrows at the ortho and para positions, the more readily the hydroxylation occurs, resulting in higher yields of $P$ CHQs/P-CBQs and then the stronger CL production by the corresponding TCPs. Therefore, based on the number of the dashed arrows, the order of CL emission for the three TCPs should be 2,3,5-TCP > 2,4,5-TCP > 2,4,6-TCP, which is in good agreement with the experimental results (Figure 1).

A Good Correlation Was Also Observed between CL Emission and the Formation of the Chlorinated $p$ Semiquinone Radicals Which Were Unequivocally Identified under the Same Experimental Conditions for CL Generation. As discussed above, $P$-CHQs and $P$-CBQs were found to be the major quinoid intermediates during the degradation of TCPs by the Fenton reagent, which are consistent with previous reports of TCPs oxidation by other
AOPs. ${ }^{41}$ It is well-known that the cyclic (auto)oxidation and reduction reactions of $P$-CHQs and $P$-CBQs can produce the intermediary of $P$-CSQs ${ }^{\bullet}{ }^{1,32}$ However, to our knowledge, there was no evidence of $P$-CSQ $s^{\bullet}$ formation during the degradation of $\mathrm{CPs}$ by the Fenton reagent, although $P$-CSQs ${ }^{\circ}$ can be detected in other ${ }^{\circ} \mathrm{OH}$-generating systems. ${ }^{42-44}$ The reason is possibly due to the rapid formation and decay of $P$-CSQs during the high yields of ${ }^{\bullet} \mathrm{OH}$ in the Fenton system. To solve this problem, a stopped-flow technique, which is suitable for the kinetic study of fast reactions, was employed. Using this method, we successfully observed the typical UV-visible spectra of $P$-CSQs ${ }^{\bullet}$ generation from the TCPs/Fenton system under the real CL-producing experimental conditions (Figure 5). As expected, these P-CSQs ${ }^{\bullet}$ were fleetingly formed and
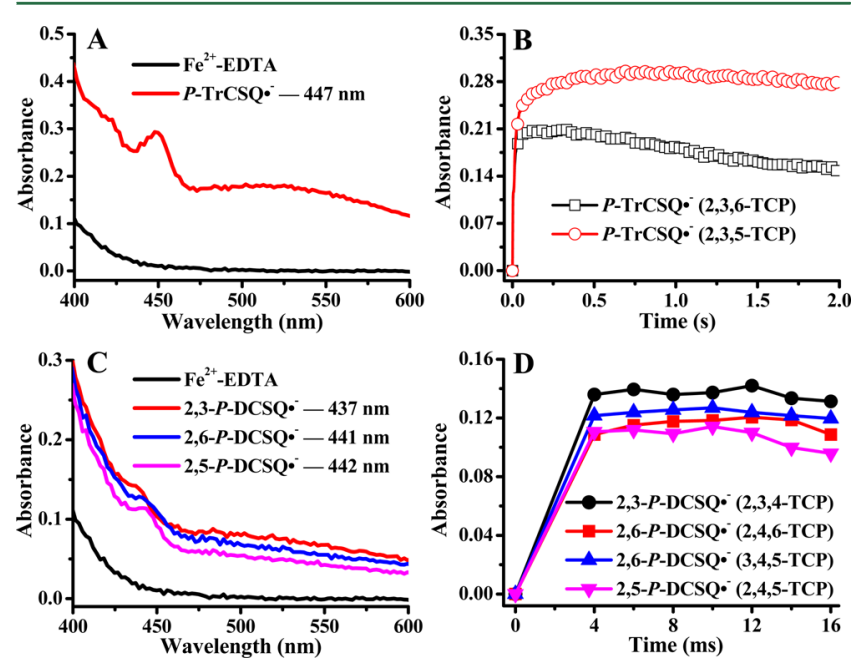

Figure 5. Transient $P$-CSQs ${ }^{\bullet}$ production by the TCPs/Fenton system under the same experimental conditions for CL generation. (A and C) Transient absorption spectra of $P$-TrCSQ ${ }^{\bullet}$ and $P$-DCSQ $s^{\circ}$, respectively. (B and D) The kinetics of $P$-TrCSQ (at $447 \mathrm{~nm}$ ) and $P$-DCSQs ${ }^{\circ}\left(2,3\right.$-dichloro- $p$-semiquinone radical $\left(2,3-P\right.$-DCSQ $\left.{ }^{\bullet}\right)$ at 437 $\mathrm{nm}, 2,6-P$-DCSQ ${ }^{\bullet}$ at $441 \mathrm{~nm}, 2,5$-dichloro- $p$-semiquinone radical $(2,5$ $P$-DCSQ $\left.{ }^{\circ}\right)$ at $442 \mathrm{~nm}$ ) formation, respectively. TCPs, $2 \mathrm{mM} ; \mathrm{H}_{2} \mathrm{O}_{2}$, $100 \mathrm{mM} ; \mathrm{Fe}^{2+}$-EDTA, $1.2 \mathrm{mM}$. All reactions were carried out in the chelex-pretreated phosphate buffer $(0.1 \mathrm{M}, \mathrm{pH} 7.4)$ at $25{ }^{\circ} \mathrm{C}$.

rapidly decayed. For example, the time of the maximum yield/ disappearance of $P$-CSQs ${ }^{\bullet}$ produced by TCPs was $0.01 \mathrm{~s} / 0.018$ $\mathrm{s}$ for 2,3,4-TCP, $0.01 \mathrm{~s} / 0.016 \mathrm{~s}$ for 2,4,6-TCP, $0.01 \mathrm{~s} / 0.016 \mathrm{~s}$ for 3,4,5-TCP, $0.01 \mathrm{~s} / 0.02 \mathrm{~s}$ for 2,4,5-TCP, $0.23 \mathrm{~s} / 2 \mathrm{~s}$ for 2,3,6$\mathrm{TCP}$, and $0.8 \mathrm{~s} / 3.9 \mathrm{~s}$ for 2,3,5-TCP, respectively.

After lowering the concentration of the Fenton reagent, $P$ $\mathrm{CSQs}^{\bullet}$ formation could also be detected by the conventional UV-visible method in the TCPs/Fenton system (Figure S4). A 
good correlation was also observed between $P$-CSQs ${ }^{\bullet}$ formation and ${ }^{\bullet} \mathrm{OH}$ production by the Fenton system (as measured by the fluorescent method with terephthalic acid (TPA) as an ${ }^{\bullet} \mathrm{OH}$ probe): the more ${ }^{\bullet} \mathrm{OH}$ produced, the faster the formation of trichloro- $p$-semiquinone radical $\left(P\right.$-TrCSQ $\left.{ }^{\bullet}\right)$ (2,3,5-TCP as a typical example) (Figure S5). However, 2,6dichloro- $p$-semiquinone radical $\left(2,6-P\right.$-DCSQ $\left.{ }^{\bullet}\right)$ produced by $2,4,6-/ 3,4,5-\mathrm{TCP}$ in the Fenton system could not be detected by the conventional UV-visible method, possibly due to the poor responses of 2,6-P-DCSQ', and the interference of absorption spectra of $\mathrm{Fe}^{2+}$-EDTA and the hydroxylated quinones. Therefore, false responses were observed on the kinetics of 2,6-P-DCSQ ${ }^{\bullet}$ formation by $2,4,6-/ 3,4,5-\mathrm{TCP}$ in the Fenton system (Figure 6B and S6A).
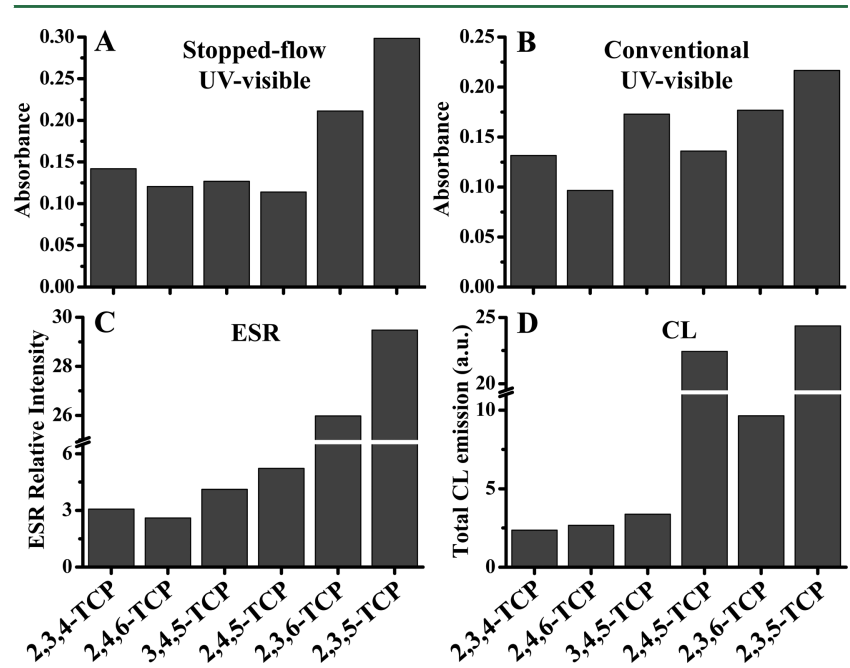

Figure 6. A correlation was observed between the CL emission and $P$ $\mathrm{CSQs}^{\circ}$ formation in the TCPs/Fenton system. (A) The yields of $P$ $\mathrm{CSQs}^{\bullet}$ by the stopped-flow technique. TCPs, $2 \mathrm{mM} ; \mathrm{H}_{2} \mathrm{O}_{2}, 100 \mathrm{mM}$; $\mathrm{Fe}^{2+}$-EDTA, $1.2 \mathrm{mM}$. (B) The yields of $P$-CSQs ${ }^{\bullet}$ by the UV-visible method. TCPs, $0.5 \mathrm{mM} ; \mathrm{H}_{2} \mathrm{O}_{2}, 0.5 \mathrm{mM}$; $\mathrm{Fe}^{2+}$-EDTA, $1.2 \mathrm{mM}$. (C) The yields of $P$-CSQs ${ }^{\bullet}$ by the direct ESR method. TCPs, $0.5 \mathrm{mM}$; $\mathrm{H}_{2} \mathrm{O}_{2}, 0.5 \mathrm{mM}$; $\mathrm{Fe}^{2+}$-EDTA, $1.2 \mathrm{mM}$. (D) The trend of CL emission from TCPs. TCPs, $0.03 \mathrm{mM} ; \mathrm{H}_{2} \mathrm{O}_{2}, 100 \mathrm{mM}$; Fe ${ }^{2+}$-EDTA, $1 \mathrm{mM}$. The reactions were carried out in the chelex-pretreated phosphate buffer $(0.1 \mathrm{M}, \mathrm{pH} 7.4)$ at $25^{\circ} \mathrm{C}$.

To further confirm whether 2,6-P-DCSQ ${ }^{\bullet}$ and other semiquinone radical intermediates were formed or not, the radicals produced in the TCPs/Fenton system were measured by the direct ESR method. All $P$-CSQs ${ }^{\bullet}$ including 2,6-P-DCSQ were found to be indeed detected and unequivocally identified by direct ESR under the same experimental conditions as that measured by the UV-visible method (Table 1). Meanwhile, based on the formation of the same $P$-CSQs ${ }^{\bullet}$ intermediates $(P$ $\mathrm{TrCSQ}^{\bullet}$ and three different dichloro- $p$-semiquinone radicals $\left(P\right.$-DCSQ $\left.\left.s^{\bullet}\right)\right)$, TCPs can also be classified into four subgroups, which agree well with the above proposed four subgroups based on the formation of the same $p$-quinoid intermediates ( $P$ CHQs $/ P$-CBQs $)$. For example, both $P$-TrCSQ $\left(a^{\mathrm{H}}=2.17 \mathrm{G}, g\right.$ $\left.=2.0051 ; \lambda_{\max }=447 \mathrm{~nm}\right)$ and $P-\mathrm{TrCHQ} / P-\mathrm{TrCBQ}$ can be produced by 2,3,5-TCP and 2,3,6-TCP, and both 2,6-P-DCSQ $\left(a^{\mathrm{H}}=2.40 \mathrm{G}, g=2.0049 ; \lambda_{\max }=441 \mathrm{~nm}\right)$ and $2,6-P-\mathrm{DCHQ} /$ 2,6-P-DCBQ can be produced by $2,4,6-\mathrm{TCP}$ and 3,4,5-TCP. Subsequently, the kinetics of $P$-CSQs $s^{\bullet}$ formation and decay in the TCPs/Fenton system were investigated by both UVvisible and direct ESR spectrometric methods (Figure S6), and
Table 1. ESR Spectra and $g$ Values of P-CSQ ${ }^{\bullet}$ Produced during the Degradation of TCPs with the Fenton Reagent

\begin{tabular}{|c|c|c|c|c|}
\hline TCPs & $\begin{array}{c}\text { ESR } \\
\text { Spectrogram }\end{array}$ & P-CSQs ${ }^{\circ-}$ & $a^{\mathbf{H}}$ & $g$ \\
\hline $\begin{array}{l}2,3,5-\mathrm{TCP} \\
2,3,6-\mathrm{TCP}\end{array}$ & $-W^{10 G}$ & $P$-TrCSQ ${ }^{\circ-}$ & 2.17 & 2.0051 \\
\hline 2,4,5-ТCP & $-1 / p$ & 2,5-P-DCSQ ${ }^{\circ-}$ & 1.96 & 2.0049 \\
\hline $\begin{array}{l}3,4,5-\mathrm{TCP} \\
2,4,6-\mathrm{TCP}\end{array}$ & $-1 / p$ & 2,6-P-DCSQ & 2.40 & 2.0049 \\
\hline 2,3,4-ТCP & $-1 / n-$ & 2,3-P-DCSQ ${ }^{\circ-}$ & 2.35 & 2.0050 \\
\hline
\end{tabular}

the maximum yields of each $P$-CSQs ${ }^{\bullet}$ were summarized (Figure $6 \mathrm{~B}$ and $6 \mathrm{C})$. It is worth noting that, due to the difference in ESR responses of the various $P$-CSQ $s^{\bullet}$, the signal intensity of $P$ TrCSQ $^{\bullet}$ produced by 2,3,5- and 2,3,6-TCP was far stronger than that of $P$-DCSQs ${ }^{\bullet}$ produced by other four TCPs under the same experimental conditions (Figure 6C and S6B). In spite of this, a pretty good correlation could also be observed between the maximum yields of $P$-CSQs $s^{\bullet}$ and the general trend of CL produced by the corresponding TCP (Figure 6), especially for those TCPs which can produce the same $P$-CHQs/P-CBQs.

In summary, these results demonstrate that the SAR for CL is closely correlated not only to the formation of the quinoid transient product but also to that of the chlorinated semiquinone radical intermediates.

Molecular Mechanism of ${ }^{\bullet} \mathrm{OH}-D e p e n d e n t \mathrm{CL}$ Production by the Highly Chlorinated Phenols and the Fenton Reagent. On the basis of all the above results and our previous findings, ${ }^{1,17-20,45-47}$ a molecular mechanism for ${ }^{\bullet} \mathrm{OH}$-dependent $\mathrm{CL}$ production by TCPs $/ \mathrm{H}_{2} \mathrm{O}_{2} / \mathrm{Fe}^{2+}$-EDTA (2,3,5-TCP was chosen as a model TCP which shows the strongest $\mathrm{CL}$ emission among the 6 TCPs) (Scheme S2) was proposed: ${ }^{\bullet} \mathrm{OH}$ first attacks TCPs, via electrophilic addition (pathways I, III, and IV) and/or electron transfer (pathway II), forming the initial chloroquinoid intermediates $(P$-CHQs, $P$-CBQs, and $O$ $\mathrm{CHQs})$ and radical intermediates ( $P$-CSQs ${ }^{\bullet}$ and chlorophenoxyl radicals $\left.\left(\mathrm{ClPhO}^{\bullet}\right)\right)$. Similar to what we proposed recently, $^{20}$ the chloroquinoid intermediates (such as $P$ TrCBQ) may further react with $\mathrm{H}_{2} \mathrm{O}_{2}$ (and/or ${ }^{\bullet} \mathrm{OH}$ ), forming a quinone-hydroperoxide reaction intermediate $P$-DCBQ$\mathrm{OOH}$, which could decompose homolytically to produce ' $\mathrm{OH}$ and the oxygen-centered enoxy radical P-DCBQ-O ${ }^{\bullet}$. $P$ $\mathrm{DCBQ}^{\bullet}$ then could either disproportionate to form the initial transient intermediate $P$-DCBQ- $\mathrm{O}^{-}$or isomerize to form the carbon-centered ketoxy radical ${ }^{\circ} \mathrm{P}-\mathrm{DCBQ}=\mathrm{O}$, which could be attacked by ${ }^{\circ} \mathrm{OH}$ to form a multicarbonyl compound (Scheme S3). The multicarbonyl compound is an unstable $o$ quinone and may further react with $\mathrm{H}_{2} \mathrm{O}_{2}$ (and/or ${ }^{\bullet} \mathrm{OH}$ ) to form a highly energetic quinone-1,2-dioxetane. An alternative pathway might be that the nucleophilic attack of $\mathrm{H}_{2} \mathrm{O}_{2}$ may preferentially occur on the $o$-quinone intermediates like $O$ CBQs, specifically on the carbonyl carbon which is supposed to be the most electrophilic one. A subsequent intramolecular nucleophilic $\mathrm{H}_{2} \mathrm{O}_{2}$ attack would give rise to a dioxetane intermediate similar to that shown in Scheme S2 but with different substitution patterns. If the quinone ring were 
substituted by more electron-withdrawing groups $(-\mathrm{Cl})$, the chloroquinoid intermediates will be more readily attacked by the nucleophile $\mathrm{H}_{2} \mathrm{O}_{2}$, due to the decrease in the $\pi$-electron density of the quinone ring. During the CL reaction, TCPs with different chemical structures can produce different quinone-1,2dioxetanes, which will further generate distinct CL emission. In general, CPs with position-2, -3 , or -5 chloro-substitution could produce the higher chlorination quinoid intermediates which will be more readily attacked by the nucleophile $\mathrm{H}_{2} \mathrm{O}_{2}$. Therefore, CPs with position-2, -3 , or -5 chloro-substitutions could lead to higher efficiency of excited state formation and then generate stronger CL. The highly energetic quinone-1,2dioxetanes further decompose to form the electronically excited carbonyl species [L]*. The CL is emitted when the electronically excited state of $[\mathrm{L}]^{*}$ returns to its ground state $[\mathrm{L}]$, and the subsequent decomposition of [L] leads to the formation of the final ring-opened products such as dichloromaleic acid (DCMA), chloromalonic acid (CMA), oxalic acid (OA), formic acid (FA), and $\mathrm{CO}_{2}$ (and/or CO) (Figure S7).

Potential Environmental Implications of SAR for CL. TCPs have been widely applied as pesticides, wood preservatives, fungicides, and as precursors in the synthesis of the widely used chlorophenoxyacetate herbicides. ${ }^{7}$ For example, 2,4,5-trichlorophenol (2,4,5-TCP) has been used as an intermediate in the manufacture of the widely used herbicide 2,4,5-trichlorophenoxyacetic acid (2,4,5-T) and the fungicide hexachlorophene. ${ }^{48}$ In Japan, since the 1970s, 2,4,6-trichlorophenol $(2,4,6-\mathrm{TCP})$ has been used as a wood preservative instead of PCP (the most toxic representative of the CPs). ${ }^{49}$ Furthermore, 2,4,5-TCP and 2,4,6-TCP are also used for the preservation of leather and textile goods. They are also found in the emissions from municipal waste incineration, landfill leachate, river water, kraft paper mill effluents, and even in chlorine-disinfected drinking water. ${ }^{7,50,51}$ During the degradation and manufacturing processes of TCPs, notorious polychlorinated dibenzo-p-dioxins and dibenzofurans (PCDD/Fs) for their carcinogenic, teratogenic, and mutagenic effects are formed. ${ }^{36,52,53}$ Consequently, it is important to detect and measure these ubiquitous and highly toxic polychlorinated phenols both in the environment and in biological systems. It is well-known that SAR approaches have been widely used to generate predicted toxicity/degradation rate data efficiently and to study the mechanism of toxicity and degradation. ${ }^{39,54-60}$ Therefore, the SAR for CL production by the CPs/Fenton system might provide useful information for predicting the toxicity/degradation rate of CPs.

Although a number of studies have been performed on quantitative structure-activity relationships (QSARs) for predicting the toxicity ${ }^{54-57}$ and degradation rate ra,58-60 of $^{39}$ CPs, there was no report on SAR between CL and the chemical structure of CPs, especially its underlying molecular mechanism. In this study, we found that the trend of CL production by all $19 \mathrm{CPs}$ and the Fenton reagent was influenced significantly by both the number and the position of the chlorine atoms on the phenolic ring. Subsequently SAR between CL and the chemical structures of the highly chlorinated phenols was established.

It has been found previously that the toxicity of chlorosubstituted phenols to various living organisms also significantly depends on the number and position of the chlorine atoms in the aromatic ring. ${ }^{55-57}$ We also observed similar results on SAR between the acute toxicity of CPs to Photobacterium phosphoreum and their chemical structure (Table S4): (i) the toxicity of CPs increased with an increasing number of chlorine atoms; (ii) for CP isomers, the toxicity decreased in the order of non- < mono- < di-ortho-chlorophenols (i.e., 3-/4-/5- > 2-/ 6- chloro-substituted phenols).

In addition, there were many studies ${ }^{39,58,60}$ on the relationship between the number and position of chlorine substituents and the degradation rate of $\mathrm{CPs}$ by ${ }^{\circ} \mathrm{OH}$ : the degradation rate constants decreased with the increase of the number of chlorine atoms on the aromatic ring, especially for the highly chlorinated phenols, whereas in the $\mathrm{CP}$ isomers, the reduced degradation rate in the order of 3-/5- > 2-/4-/6- chloro-substituted phenols.

Comparing our study with the previous studies ${ }^{39,54,55}$ on SARs between the toxicity/degradation rate and the chemical structure of CPs, we found there are some good correlations between CL and the toxicity/degradation rate of CPs (although there are some exceptions): (i) In general, CPs exhibit the stronger CL, higher toxicity, and slower degradation rate with an increasing number of chlorine atoms on the phenolic ring; (ii) For each isomeric group (Table S5), CPs with position-3 or/and -5 substitutions, such as 3-CP, 3,5-DCP, 3,4,5-TCP, and 2,3,4,5-TeCP, exhibit relatively stronger CL, higher toxicity, and faster degradation rate compared to other corresponding isomers; whereas those with position-6 substituents, such as 2,6-DCP, 2,4,6-TCP, and 2,3,4,6-TeCP, possess the weakest $\mathrm{CL}$, the lowest toxicity, and the slowest degradation rate. These correlations may be due to similar or even the same dominating physiochemical factors (as discussed above, Table S6) such as the directing effect, intramolecular hydrogen bonding, and steric hindrance effect of $-\mathrm{OH} /-\mathrm{Cl}$ groups, as well as the electron-withdrawing properties of $\mathrm{Cl}$ atoms.

Although SAR between CL and their chemical structure shows great promise for the prediction of the toxicity and degradability of CPs, the prediction model also shows several limitations. The main limitation involves the low predictability for 2-/4- substituted polychlorinated phenols. This might be attributed to the fact that the underlying molecular mechanisms for the CL production, toxicity, and degradation rate of CPs are not exactly the same (Table S6). Because of these reasons, the effects of position- 2 and -4 on CL, the toxicity, and degradation rate of CPs are not always consistent with each other. For example, the CL of 2,4,5-TCP is far stronger than that of 2,3,4TCP, but the toxicity and degradation rate of the two TCPs are equivalent. Another limitation is that until now, the toxicity assessment is limited only to polychlorinated phenols but not to other halogenated $(-\mathrm{F},-\mathrm{Br})$ phenols and even derivatives of other polyhalogenated aromatic compounds. Further investigations on the derivatives of phenol by this CL technique are needed for our future studies.

In summary, we found that for the SAR for CL of all $19 \mathrm{CPs} /$ Fenton systems, the CL increased with an increasing number of chlorine atoms in general; and for $\mathrm{CP}$ isomers, the $\mathrm{CL}$ decreased in the order of 2-/3-/5- > 4-/6-Cl-substituents. During the degradation of TCPs by the Fenton reagent, the chlorinated quinoid intermediates and semiquinone radicals were produced, and the type and yield of which were well correlated with their CL generation. This study is the first report on the SAR study for CL and the critical role of quinoid intermediates and semiquinone radicals in SAR. Meanwhile, the fact that CL could be produced by CPs and the Fenton reagent may imply that this unique CL-producing property can be used to detect and measure CPs, an important class of compounds of major environmental and biomedical concern. Indeed, we took 
advantage of this finding and employed this highly sensitive CL method to measure quantitatively trace amounts of CPs and found that the detection limits of TCPs in the Fenton system are low and linear ranges are wide (Table S7). On the basis of the above results, this novel CL-dependent method can be used not only to detect and measure trace amounts of CPs in both pure and real samples but also to provide useful information for predicting the toxicity/degradation rate of CPs. Therefore, these new findings may have broad chemical and environmental implications for future studies on remediation of other halogenated persistent organic pollutants by advanced oxidation processes.

\section{ASSOCIATED CONTENT}

\section{S Supporting Information}

The Supporting Information is available free of charge on the ACS Publications website at DOI: 10.1021/acs.est.6b04664.

Additional experimental details and figures (PDF)

\section{AUTHOR INFORMATION}

\section{Corresponding Author}

*Phone: +86-10-62849030. Fax: +86-10-62923563. E-mail: bzhu@rcees.ac.cn.

\section{ORCID}

Ben-Zhan Zhu: 0000-0001-5484-4290

\section{Notes}

The authors declare no competing financial interest.

\section{ACKNOWLEDGMENTS}

The work in this paper was supported by SPRP CAS (XDB01020300); NSFC Grants (21577149, 21477139, 21237005, 21621064, 21407163, and 21321004); National Institutes of Health Grants ES11497, RR01008, and ES00210; and the Fundamental Research Funds for the Central Universities (2014MS155).

\section{REFERENCES}

(1) Zhu, B. Z.; Shan, G. Q. Potential mechanism for pentachlorophenol-induced carcinogenicity: A novel mechanism for metalindependent production of hydroxyl radicals. Chem. Res. Toxicol. 2009, 22, 969-977.

(2) Zhu, B. Z.; Zhu, J. G.; Fan, R. M.; Mao, L. Metal-independent pathways of chlorinated phenol/quinone toxicity. Adv. Mol. Toxicol. 2011, 5, 1-43.

(3) Gupta, S. S.; Stadler, M.; Noser, C. A.; Ghosh, A.; Steinhoff, B.; Lenoir, D.; Horwitz, C. P.; Schramm, K. W.; Collins, T. J. Rapid total destruction of chlorophenols by activated hydrogen peroxide. Science 2002, 296, 326-328.

(4) Pera-Titus, M.; Garcia-Molina, V.; Banos, M. A.; Gimenez, J.; Esplugas, S. Degradation of chlorophenols by means of advanced oxidation processes: a general review. Appl. Catal., B 2004, 47, 219256.

(5) U. S. Environmental Protection Agency. National recommended water quality criteria; 2004. https://www.epa.gov/sites/production/ files/2015-06/documents/nrwqc-2004.pdf (accessed Feb 3, 2017).

(6) International Agency for Research on Cancer (IARC), Pentachlorophenol. IARC monographs on the evaluation of carcinogenic risks to humans: Occupational exposures in insecticide application, and some pesticides, Volume 53; 1991. http:// monographs.iarc.fr/ENG/Monographs/vol53/mono53-15.pdf (accessed Feb 3, 2017).

(7) Czaplicka, M. Sources and transformations of chlorophenols in the natural environment. Sci. Total Environ. 2004, 322, 21-39.
(8) Teel, A. L.; Warberg, C. R.; Atkinson, D. A.; Watts, R. J. Comparison of mineral and soluble iron Fenton's catalysts for the treatment of trichloroethylene. Water Res. 2001, 35, 977-984.

(9) Kwan, W. P.; Voelker, B. M. Decomposition of hydrogen peroxide and organic compounds in the presence of dissolved iron and ferrihydrite. Environ. Sci. Technol. 2002, 36, 1467-1476.

(10) Schuster, G. B. Chemiluminescence of organic peroxides. Conversion of ground-state reactants to excited-state products by the chemically initiated electron-exchange luminescence mechanism. Acc. Chem. Res. 1979, 12, 366-373.

(11) Matsumoto, M. Advanced chemistry of dioxetane-based chemiluminescent substrates originating from bioluminescence. $J$. Photochem. Photobiol., C 2004, 5, 27-53.

(12) Widder, E. A. Bioluminescence in the ocean: origins of biological, chemical, and ecological diversity. Science 2010, 328, 704708.

(13) Adam, W.; Kazakov, D. V.; Kazakov, V. P. Singlet-oxygen chemiluminescence in peroxide reactions. Chem. Rev. 2005, 105, 3371-3387.

(14) Grayeski, M. L. Chemiluminescence analysis. Anal. Chem. 1987, 59, 1243A-1256A.

(15) Das, S.; Powe, A. M.; Baker, G. A.; Valle, B.; El-Zahab, B.; Sintim, H. O.; Lowry, M.; Fakayode, S. O.; McCarroll, M. E.; Patonay, G. Molecular fluorescence, phosphorescence, and chemiluminescence spectrometry. Anal. Chem. 2012, 84, 597-625.

(16) Liu, M. L.; Lin, Z.; Lin, J. M. A review on applications of chemiluminescence detection in food analysis. Anal. Chim. Acta 2010, $670,1-10$

(17) Zhu, B. Z.; Zhao, H. T.; Kalyanaraman, B.; Liu, J.; Shan, G. Q.; Du, Y. G.; Frei, B. Mechanism of metal-independent decomposition of organic hydroperoxides and formation of alkoxyl radicals by halogenated quinones. Proc. Natl. Acad. Sci. U. S. A. 2007, 104, 3698-3702.

(18) Zhu, B. Z.; Kalyanaraman, B.; Jiang, G. B. Molecular mechanism for metal-independent production of hydroxyl radicals by hydrogen peroxide and halogenated quinones. Proc. Natl. Acad. Sci. U. S. A. 2007, 104, 17575-17578.

(19) Zhu, B. Z.; Shan, G. Q.; Huang, C. H.; Kalyanaraman, B.; Mao, L.; Du, Y. G. Metal-independent decomposition of hydroperoxides by halogenated quinones: Detection and identification of a quinone ketoxy radical. Proc. Natl. Acad. Sci. U. S. A. 2009, 106, 11466-11471.

(20) Zhu, B. Z.; Mao, L.; Huang, C. H.; Qin, H.; Fan, R. M.; Kalyanaraman, B.; Zhu, J. G. Unprecedented hydroxyl radicaldependent two-step chemiluminescence production by polyhalogenated quinoid carcinogens and $\mathrm{H}_{2} \mathrm{O}_{2}$. Proc. Natl. Acad. Sci. U. S. A. 2012, 109, 16046-16051.

(21) Huang, C. H.; Shan, G. Q.; Mao, L.; Kalyanaraman, B.; Qin, H.; Ren, F. R.; Zhu, B. Z. The first purification and unequivocal characterization of the radical form of the carbon-centered quinone ketoxy radical adduct. Chem. Commun. 2013, 49, 6436-6438.

(22) Qin, H.; Huang, C. H.; Mao, L.; Xia, H. Y.; Kalyanaraman, B.; Shao, J.; Shan, G. Q.; Zhu, B. Z. Molecular mechanism of metalindependent decomposition of lipid hydroperoxide 13-HPODE by halogenated quinoid carcinogens. Free Radical Biol. Med. 2013, 63, 459-466.

(23) Huang, C. H.; Ren, F. R.; Shan, G. Q.; Qin, H.; Mao, L.; Zhu, B. Z. Molecular mechanism of metal-independent decomposition of organic hydroperoxides by halogenated quinoid carcinogens and the potential biological implications. Chem. Res. Toxicol. 2015, 28, 831837.

(24) Yin, R.; Zhang, D.; Song, Y.; Zhu, B. Z.; Wang, H. Potent DNA damage by polyhalogenated quinones and $\mathrm{H}_{2} \mathrm{O}_{2}$ via a metalindependent and intercalation-enhanced oxidation mechanism. Sci. Rep. 2013, 3, 1-6.

(25) Shao, J.; Huang, C. H.; Kalyanaraman, B.; Zhu, B. Z. Potent methyl oxidation of 5-methyl-2'-deoxycytidine by halogenated quinoid carcinogens and hydrogen peroxide via a metal-independent mechanism. Free Radical Biol. Med. 2013, 60, 177-182. 
(26) Jia, S.; Zhu, B. Z.; Guo, L. H. Detection and mechanistic investigation of halogenated benzoquinone induced DNA damage by photoelectrochemical DNA sensor. Anal. Bioanal. Chem. 2010, 397, 2395-2400.

(27) Mao, L.; Liu, Y. X.; Huang, C. H.; Gao, H. Y.; Kalyanaraman, B.; $\mathrm{Zhu}, \mathrm{B}$. Z. Intrinsic chemiluminescence generation during advanced oxidation of persistent halogenated aromatic carcinogens. Environ. Sci. Technol. 2015, 49, 7940-7947.

(28) Usman, M.; Hanna, K.; Haderlein, S. Fenton oxidation to remediate PAHs in contaminated soils: A critical review of major limitations and counter-strategies. Sci. Total Environ. 2016, 569-570, 179-190.

(29) He, J.; Yang, X. F.; Men, B.; Wang, D. S. Interfacial mechanisms of heterogeneous Fenton reactions catalyzed by iron-based materials: A review. J. Environ. Sci. (Beijing, China) 2016, 39, 97-109.

(30) Yang, X. F.; Guo, X. Q. Fe(II)-EDTA chelate-induced aromatic hydroxylation of terephthalate as a new method for the evaluation of hydroxyl radical-scavenging ability. Analyst 2001, 126, 928-932.

(31) Duling, D. R. Simulation of multiple isotropic spin-trap EPR spectra. J. Magn. Reson., Ser. B 1994, 104, 105-110.

(32) Bolton, J. L.; Trush, M. A.; Penning, T. M.; Dryhurst, G.; Monks, T. J. Role of quinones in toxicology. Chem. Res. Toxicol. 2000, 13, 135-160.

(33) Isaacs, N. S.; van Eldik, R. A mechanistic study of the reduction of quinones by ascorbic acid. J. Chem. Soc., Perkin Trans. 2 1997, $1465-1467$.

(34) Pethig, R.; Gascoyne, P. R.; McLaughlin, J. A.; Szent-Gyorgyi, A. Ascorbate-quinone interactions: Electrochemical, free radical, and cytotoxic properties. Proc. Natl. Acad. Sci. U. S. A. 1983, 80, 129-132.

(35) Goldstein, S.; Meyerstein, D.; Czapski, G. The Fenton reagents. Free Radical Biol. Med. 1993, 15, 435-445.

(36) Czaplicka, M. Photo-degradation of chlorophenols in the aqueous solution. J. Hazard. Mater. 2006, 134, 45-59.

(37) Carey, F. A. Organic chemistry, 4th ed.; McGraw-Hill Higher Education: U.S.A., 2000; pp 463-472.

(38) Akai, N.; Kudoh, S.; Takayanagi, M.; Nakata, M. Photoreaction mechanisms of 2-chlorophenol and its multiple chloro-substituted derivatives studied by low-temperature matrix-isolation infrared spectroscopy and density-functional-theory calculations. J. Photochem. Photobiol., A 2001, 146, 49-57.

(39) Tang, W. Z.; Huang, C. P. The effect of chlorine position of chlorinated phenols on their dechlorination kinetics by Fenton's reagent. Waste Manage. 1995, 15, 615-622.

(40) Kishino, T.; Kobayashi, K. Relation between the chemical structures of chlorophenols and their dissociation constants and partition coefficients in several solvent-water systems. Water Res. 1994, $28,1547-1552$.

(41) Sharma, S.; Mukhopadhyay, M.; Murthy, Z. V. P. Treatment of chlorophenols from wastewaters by advanced oxidation processes. Sep. Purif. Rev. 2013, 42, 263-295.

(42) Han, S. K.; Ichikawa, K.; Utsumi, H. Generation of $p$ semiquinone radicals from chlorophenols in water during ozonation. Water Res. 1998, 32, 1978-1981.

(43) Zimbron, J. A.; Reardon, K. F. Fenton's oxidation of pentachlorophenol. Water Res. 2009, 43, 1831-1840.

(44) Hong, P. K.; Zeng, Y. Degradation of pentachlorophenol by ozonation and biodegradability of intermediates. Water Res. 2002, 36, $4243-4254$.

(45) Zhu, B. Z.; Zhao, H. T.; Kalyanaraman, B.; Frei, B. Metalindependent production of hydroxyl radicals by halogenated quinones and hydrogen peroxide: An ESR spin trapping study. Free Radical Biol. Med. 2002, 32, 465-473.

(46) Zhu, B. Z.; Zhu, J. G.; Mao, L.; Kalyanaraman, B.; Shan, G. Q. Detoxifying carcinogenic polyhalogenated quinones by hydroxamic acids via an unusual double Lossen rearrangement mechanism. Proc. Natl. Acad. Sci. U. S. A. 2010, 107, 20686-20690.

(47) Shan, G. Q.; Yu, A.; Zhao, C. F.; Huang, C. H.; Zhu, L. Y.; Zhu, B. Z. A combined experimental and computational investigation on the unusual molecular mechanism of the Lossen rearrangement reaction activated by carcinogenic halogenated quinones. J. Org. Chem. 2015, $80,180-189$.

(48) International Agency for Research on Cancer (IARC), IARC monographs on the evaluation of the carcinogenic risk of chemicals to humans: Some halogenated hydrocarbons and pesticide exposure. Lyon, France, Volume 41; 1986. http://monographs.iarc.fr/ENG/ Monographs/vol1-42/index.php (accessed Feb 3, 2017).

(49) Kiyohara, H.; Hatta, T.; Ogawa, Y.; Kakuda, T.; Yokoyama, H.; Takizawa, N. Isolation of Pseudomonas pickettii strains that degrade 2,4,6-trichlorophenol and their dechlorination of chlorophenols. Appl. Environ. Microbiol. 1992, 58, 1276-1283.

(50) Chen, G. C.; Shan, X. Q.; Wang, Y. S.; Wen, B.; Pei, Z. G.; Xie, Y. N.; Liu, T.; Pignatello, J. J. Adsorption of 2,4,6-trichlorophenol by multi-walled carbon nanotubes as affected by $\mathrm{Cu}(\mathrm{II})$. Water Res. 2009, 43, 2409-2418.

(51) Chu, W.; Law, C. K. Treatment of trichlorophenol by catalytic oxidation process. Water Res. 2003, 37, 2339-2346.

(52) Androulaki, E.; Hiskia, A.; Dimotikali, D.; Minero, C.; Calza, P.; Pelizzetti, E.; Papaconstantinou, E. Light induced elimination of mono- and polychlorinated phenols from aqueous solutions by $\mathrm{PW}_{12} \mathrm{O}_{40}{ }^{3-}$. The case of 2,4,6-trichlorophenol. Environ. Sci. Technol. 2000, 34, 2024-2028.

(53) Qu, X. H.; Wang, H.; Zhang, Q. Z.; Shi, X. Y.; Xu, F.; Wang, W. $X$. Mechanistic and kinetic studies on the homogeneous gas-phase formation of $\mathrm{PCDD} / \mathrm{Fs}$ from 2,4,5-trichlorophenol. Environ. Sci. Technol. 2009, 43, 4068-4075.

(54) Smith, S.; Furay, V. J.; Layiwola, P. J.; Menezes-Filho, J. A. Evaluation of the toxicity and quantitative structure-activity relationships (QSAR) of chlorophenols to the copepodid stage of a marine copepod (Tisbe battagliai) and two species of benthic flatfish, the flounder (Platichthys flesus) and sole (Solea solea). Chemosphere 1994, 28, 825-836.

(55) Padmanabhan, J.; Parthasarathi, R.; Subramanian, V.; Chattaraj, P. K. Group philicity and electrophilicity as possible descriptors for modeling ecotoxicity applied to chlorophenols. Chem. Res. Toxicol. 2006, 19, 356-364.

(56) Kishino, T.; Kobayashi, K. Acute toxicity and structure-activity relationships of chlorophenols in fish. Water Res. 1996, 30, 387-392.

(57) Chen, C. Y.; Lin, J. H. Toxicity of chlorophenols to Pseudokirchneriella subcapitata under air-tight test environment. Chemosphere 2006, 62, 503-509.

(58) Antonaraki, S.; Androulaki, E.; Dimotikali, D.; Hiskia, A.; Papaconstantinou, E. Photolytic degradation of all chlorophenols with polyoxometallates and $\mathrm{H}_{2} \mathrm{O}_{2}$. J. Photochem. Photobiol., A 2002, 148, 191-197.

(59) Parra, S.; Olivero, J.; Pacheco, L.; Pulgarin, C. Structural properties and photoreactivity relationships of substituted phenols in $\mathrm{TiO}_{2}$ suspensions. Appl. Catal., B 2003, 43, 293-301.

(60) Oturan, N.; Panizza, M.; Oturan, M. A. Cold incineration of chlorophenols in aqueous solution by advanced electrochemical process electro-Fenton. Effect of number and position of chlorine atoms on the degradation kinetics. J. Phys. Chem. A 2009, 113, 1098810993. 